\title{
Twist-3 approach to hyperon polarization in unpolarized proton-proton collision
}

\author{
Kenta Yabe* \\ Graduate School of Science and Technology, Niigata University, Ikarashi, Niigata 950-2181, \\ Japan \\ E-mail: yabe.niigata.hadron0127@gmail.com
}

\section{Yuji Koike}

Department of Physics, Niigata University, Ikarashi, Niigata 950-2181, Japan

E-mail: koike@nt.sc.niigata-u.ac.jp

\section{Shinsuke Yoshida}

Key Laboratory of Quark and Lepton Physics (MOE) and institute of Particle Physics, Central China Normal University. Wuhan 430079, China

E-mail: shinyoshida85@gmail.com

\begin{abstract}
We study the transverse polarization of hyperons produced in high-energy unpolarized protonproton collisions in the framework of the collinear factorization. This phenomenon is a twist-3 observable which receives contributions from a twist-3 distribution and a twist- 3 fragmentation function. We focus on the former contribution and derive the corresponding leading-order cross section. For the soft-gluon-pole contribution, we develop the "master formula" which reduces the partonic cross section to a certain $2 \rightarrow 2$ parton scattering cross section and thus simplifies the calculation greatly. We also calculate the soft-fermion-pole contribution for the first time and show that it vanishes in all channels.
\end{abstract}

XXIV International Workshop on Deep-Inelastic Scattering and Related Subjects

11-15 April, 2016

DESY Hamburg, Germany

\footnotetext{
* Speaker.
} 


\section{Introduction}

Transverse polarization of hyperons produced in high-energy unpolarized proton-proton collision, $p p \rightarrow \Lambda^{\uparrow} X$, has been known for a long time since its first discovery in 1970s [1]. Conventional parton model and perturbative QCD, which had a success in the description of high energy processes, could not account for this phenomenon. Therefore it has been a big challenge for QCD theorists to understand the mechanism of the polarization. This is an example of transverse single-spin asymmetries (SSA), since only one particle participating in the scattering is transversely polarized. Other observed SSAs include $p p^{\uparrow} \rightarrow \pi X$ and $e p^{\uparrow} \rightarrow e \pi X$ etc.

Nowadays it is known that SSAs are a twist-3 observable in the collinear factorization [2] [], which is valid when an observed final state hadron has a large transverse momentum, $P_{T} \sim Q \gg$ $\Lambda_{Q C D}$. In this framework, SSA is described as an effect of multi-parton correlations either in the initial nucleon or in the final fragmentation process. These effects appear in the form of a twist- 3 distribution function or a twist-3 fragmentation function in the spin-dependent cross section. So far the complete twist-3 cross section for $p^{\uparrow} p \rightarrow \pi X$ has been derived [4, 5, 6, 8, and the RHIC data for this process [10] has been analyzed [11]. However, for $p p \rightarrow \Lambda^{\uparrow} X$, complete twist-3 cross section is not yet available. In this paper, we study this process based on the twist- 3 mechanism.

For $p(p)+p\left(p^{\prime}\right) \rightarrow \Lambda^{\uparrow}\left(P_{h}, S_{\perp}\right)+X$, the twist-3 cross section consists of two terms:

$$
\sigma \sim \sum_{a, b, c} \begin{cases}(A) & E_{F}^{a}\left(x_{1}, x_{2}\right) \otimes f_{1}^{b}\left(x^{\prime}\right) \otimes H_{1}^{c}(z) \otimes \sigma_{A} \\ + & \\ (B) & f_{1}^{a}(x) \otimes f_{1}^{b}\left(x^{\prime}\right) \otimes \hat{G}_{F}^{c}\left(z_{1}, z_{2}\right) \otimes \sigma_{B},\end{cases}
$$

where the subscripts $a, b, c$ denote quark species and the symbol $\otimes$ denotes convolution with respect to the momentum fractions. Here, $E_{F}^{a}$ and $\hat{G}_{F}^{c}$ represent the twist-3 quark-gluon correlation function in the nucleon and the twist-3 fragmentation function for $\Lambda^{\uparrow}$, respectively. Other distribution and fragmentation functions are twist-2; $f_{1}^{a}$ is the unpolarized distribution function and $H_{1}^{c}$ is the transversity fragmentation function for $\Lambda^{\uparrow}$. $\sigma_{A, B}$ represent the partonic cross sections. In this study, we focus on the first term. The hyperon polarization is a naively $T$-odd observable, which occurs as an interference between the amplitudes that have different complex phases. Accordingly the hard part for the (A) term occurs as a pole contribution from an internal propagator. This pole contribution is classified into two kinds, i.e., soft-fermion-pole (SFP) which leads to $x_{1}=0$ or $x_{2}=0$, and soft-gluon-pole (SGP) which results in $x_{1}=x_{2}$. The SGP contribution appears as derivative and nonderivative terms of the SGP function $E_{F}^{a}(x, x)$. These were calculated in the previous studies [12,13] and it was shown that only the derivative term contributes. The SFP contribution appears only as a nonderivative term, but has not been calculated yet.

The purpose of this study is twofold. First we present a rederivation of the SGP cross section in the light of the "master formula" which connects the partonic hard part for the SGP cross section to a certain $2 \rightarrow 2$ parton scattering cross section. This will give a relation between the hard cross section for the derivative term and that for the nonderivative one. Second we present the calculation for the SFP contribution for the first time. 


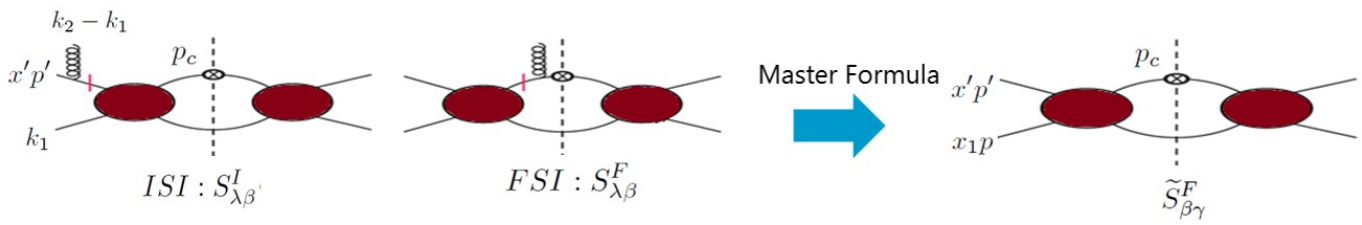

Figure 1: Diagrammatic representation of the master formula for the SGP contribution. The partonic SGP cross section corresponding to ISI and FSI can be obtained from a $2 \rightarrow 2$ partonic diagram. Each blob represents the $2 \rightarrow 2$ scattering amplitude.

\section{SGP contribution}

We consider the (A) term in 1.1]. In this contribution, a parton coming from the proton with the momentum $p^{\prime}$ has a momentum $x^{\prime} p^{\prime}$ and a quark fragmenting into the final $\Lambda^{\uparrow}$ has a momentum $p_{c}=P_{h} / z$. Twist-3 quark-gluon correlation function contributes from the proton with the momentum $p$. The SGP contribution was calculated in the previous studies [12]13] and it was shown that the non-derivative term vanishes [13], which is in contrast to the case of $p^{\uparrow} p \rightarrow \pi X$. In this section, we develop a master formula for this contribution, which has an advantage that it can simplify the actual calculation and make clear the origin of the vanishing nonderivative term. The master formula was first developed for the contribution from the twist-3 quark-gluon correlation function in the transversely polarized nucleon to $e p^{\uparrow} \rightarrow e \pi X$ [14] and $p^{\uparrow} p \rightarrow \pi X$ [15].

The SGP contribution consists of 2 types of diagram, i.e., the initial-state-interaction (ISI) diagrams and the final-state-interaction (FSI) diagrams, depending on to which parton line the coherent gluon attaches. We call the hard parts for these contributions $S_{\lambda \beta}^{\mathrm{I}, \mathrm{F}}\left(k_{1}, k_{2}, x^{\prime} p^{\prime}, p_{c}\right) p^{\lambda}$ where $k_{1}$ and $k_{2}$ are the quark momenta entering the initial proton with the momentum $p$ before collinear expansion. To get the partonic hard part for the SGP cross section, we need to calculate the derivative of $S_{\lambda \beta}^{\mathrm{I}, \mathrm{F}}$ with respect to $k_{2}$ and take the collinear limit $k_{1,2} \rightarrow x_{1,2} p$. Master formula for this contribution reads [16]

$$
\begin{aligned}
& \left.\varepsilon^{\alpha \beta n p} \frac{\partial S_{\lambda \beta}^{\mathrm{F}}\left(k_{1}, k_{2}, x^{\prime} p^{\prime}, p_{c}\right) p^{\lambda}}{\partial k_{2}^{\alpha}}\right|_{k_{i}=x_{i} p} ^{\mathrm{SGP}} \\
& =\left[\frac{1}{x_{1}-x_{2}+i \varepsilon}\right]^{\mathrm{pole}} \varepsilon^{\alpha \beta n p}\left[S_{\perp}^{\gamma} \frac{d}{d p_{c}^{\alpha}}+\frac{1}{p \cdot p_{c}}\left\{\left(p \cdot S_{\perp}\right) g_{\alpha}^{\gamma}-S_{\perp \alpha} p^{\gamma}\right\}\right] \widetilde{S}_{\beta \gamma}^{\mathrm{F}}\left(x_{1} p, x^{\prime} p^{\prime}, p_{c}\right), \\
& \left.\varepsilon^{\alpha \beta n p} \frac{\partial S_{\lambda \beta}^{\mathrm{I}}\left(k_{1}, k_{2}, x^{\prime} p^{\prime}, p_{c}\right) p^{\lambda}}{\partial k_{2}^{\alpha}}\right|_{k_{i}=x_{i} p} ^{\mathrm{SGP}}=\left[\frac{-1}{x_{2}-x_{1}+i \varepsilon}\right]^{\text {pole }} \varepsilon^{\alpha \beta n p} S_{\perp}^{\gamma} \frac{d}{d\left(x^{\prime} p^{\prime \alpha}\right)} \widetilde{S}_{\beta \gamma}^{\mathrm{I}}\left(x_{1} p, x^{\prime} p^{\prime}, p_{c}\right),(2 .
\end{aligned}
$$

where $S_{\perp}$ is the polarization vector for $\Lambda^{\uparrow}$ and $\widetilde{S}_{\lambda \beta}^{\mathrm{F}, \mathrm{I}}$ represents a certain $2 \rightarrow 2$ scattering cross section. From these formulae, the SGP cross section can be written in the following form:

$$
\begin{aligned}
E_{P_{h}} \frac{d \Delta \sigma^{\mathrm{FSI}}}{d^{3} P_{h}}= & \frac{M_{N}}{32 \pi s} \int \frac{d x^{\prime}}{x^{\prime}} f_{1}\left(x^{\prime}\right) \int \frac{d z}{z^{2}} H_{1}(z) \int \frac{d x}{x} \delta(\hat{s}+\hat{t}+\hat{u}) \varepsilon^{p_{c} p n S_{\perp}} \\
& \times\left(\frac{\hat{s}}{\hat{t} \hat{u}}\right)\left[x \frac{d E_{F}(x, x)}{d x} \Delta \sigma_{D}^{\mathrm{F}}+E_{F}(x, x)\left(-\Delta \sigma_{D}^{\mathrm{F}}+\Delta \sigma_{N D}^{\mathrm{F}}\right)\right],
\end{aligned}
$$




$$
\begin{aligned}
E_{P_{h}} \frac{d \Delta \sigma^{\mathrm{ISI}}}{d^{3} P_{h}}=-\frac{M_{N}}{32 \pi s} \int \frac{d x^{\prime}}{x^{\prime}} f_{1}\left(x^{\prime}\right) \int \frac{d z}{z^{2}} H_{1}(z) \int \frac{d x}{x} \delta(\hat{s}+\hat{t}+\hat{u}) \varepsilon^{p_{c} p n S_{\perp}} \\
\quad \times \frac{1}{\hat{u}}\left[x \frac{d E_{F}(x, x)}{d x} \Delta \sigma_{D}^{\mathrm{I}}+E_{F}(x, x)\left(-\Delta \sigma_{D}^{\mathrm{I}}+\Delta \sigma_{N D}^{\mathrm{I}}\right)\right],
\end{aligned}
$$

where $s=\left(p+p^{\prime}\right)^{2}, \hat{s}=\left(x p+x^{\prime} p^{\prime}\right)^{2}, \hat{t}=(x p-p c)^{2}, \hat{u}=\left(x^{\prime} p^{\prime}-p c\right)^{2}$ and $M_{N}$ is the nucleon mass. In (2.3) and (2.4), $\Delta \sigma_{D, N D}^{F, I}$ represents some $2 \rightarrow 2$ partonic cross sections. The appearance of $\Delta \sigma_{N D}^{F, I}$ is the different point from the case of $p^{\uparrow} p \rightarrow h X$, which is due to the fact that the spin vector $S_{\perp}$ is involved in 2.1) and 2.2. After calculation of $\Delta \sigma_{D}^{F, I}$ and $\Delta \sigma_{N D}^{F, I}$, it turned out that $\Delta \sigma_{D}^{F, I}=\Delta \sigma_{N D}^{F, I}$. This implies that the nonderivative terms vanish identically, which is consistent with what was found by direct calculation in [13]. This way we finally obtained the following result:

$$
\begin{aligned}
E_{P_{h}} & \frac{d \Delta \sigma^{\mathrm{SGP}}}{d^{3} P_{h}} \\
= & \frac{\pi M_{N} \alpha_{s}^{2}}{s} \varepsilon^{P_{h} p n S_{\perp}} \sum_{a, b, c} \int \frac{d x^{\prime}}{x^{\prime}} f_{1}^{b}\left(x^{\prime}\right) \int \frac{d z}{z^{3}} H_{1}^{c}(z) \int d x \frac{d E_{F}^{a}(x, x)}{d x} \sigma_{a b \rightarrow c} \delta(\hat{s}+\hat{t}+\hat{u}),
\end{aligned}
$$

with the partonic cross section in each channel

$$
\begin{aligned}
& \sigma_{q q^{\prime} \rightarrow q}=\frac{1}{N^{2}} \frac{2 \hat{s}}{\hat{t}^{2}}-\frac{1}{N^{2}} \frac{\hat{s}^{2}}{\hat{t}^{3}}, \quad \sigma_{q q \rightarrow q}=\sigma_{q q^{\prime} \rightarrow q}-\left(\frac{1}{N}+\frac{1}{N^{3}}\right) \frac{\hat{s}}{\hat{t} \hat{u}}+\frac{1}{N^{3}} \frac{\hat{s}^{2}}{\hat{t}^{2} \hat{u}}, \\
& \sigma_{q \bar{q}^{\prime} \rightarrow q}=\left(\frac{N^{2}-2}{N^{2}}\right) \frac{\hat{s}}{\hat{t}^{2}}-\frac{1}{N^{2}} \frac{\hat{s}^{2}}{\hat{t}^{3}}, \quad \sigma_{q \bar{q} \rightarrow q}=\sigma_{q \bar{q}^{\prime} \rightarrow q \bar{q}^{\prime}}+\frac{1}{N^{3}} \frac{1}{t}+\frac{1}{N^{3}} \frac{\hat{s}}{\hat{t}^{2}}, \\
& \sigma_{q \bar{q} \rightarrow \bar{q}}=-\frac{1}{N^{3}} \frac{1}{\hat{u}}+\left(\frac{1}{N}+\frac{1}{N^{3}}\right) \frac{\hat{s}}{\hat{t} \hat{u}}, \\
& \sigma_{q g \rightarrow q}=-\frac{N^{2}}{N^{2}-1} \frac{\hat{u}}{t^{2}}+\frac{1}{N^{2}-1} \frac{1}{\hat{u}}-\frac{1}{N^{2}\left(N^{2}-1\right)} \frac{\hat{s}}{\hat{t} \hat{u}}-\frac{1}{\left(N^{2}-1\right)} \frac{2 \hat{s}^{2}}{\hat{t}^{3}},
\end{aligned}
$$

where $N=3$ is the number of colors for a quark. These results also agree with the previous ones [12]13].

\section{SFP contribution}

According to the twist-3 formalism of [3], the SFP cross section for this process can be obtained from the following formula:

$$
\begin{aligned}
E_{P_{h}} \frac{d \Delta \sigma}{d^{3} P_{h}}= & \frac{i M_{N}}{64 \pi^{2} s} \int \frac{d x^{\prime}}{x^{\prime}} f_{1}\left(x^{\prime}\right) \int \frac{d z}{z^{2}} H_{1}(z) \\
& \times \int d x_{1} \int d x_{2} E_{F}\left(x_{1}, x_{2}\right) \varepsilon^{\alpha \beta n p}\left(\frac{1}{x_{1}-x_{2}}\right) S_{\alpha \beta}^{\mathrm{SFP}}\left(x_{1} p, x_{2} p\right),
\end{aligned}
$$

where $S_{\alpha \beta}^{\mathrm{SFP}}$ denotes the hard part for the SFP contribution. By this formula, we calculate the partonic diagrams shown in Figs. 2-4. It turned out that the SFP partonic cross section vanishes identically in all channels after summing over all diagrams. This differs from the case of $p^{\uparrow} p \rightarrow$ $\pi X$ [7] where the SFP cross section survives. From this result the SGP contribution given in 2.5] is the only contribution from the twist-3 unpolarized distribution to $p p \rightarrow \Lambda^{\uparrow} X$. 


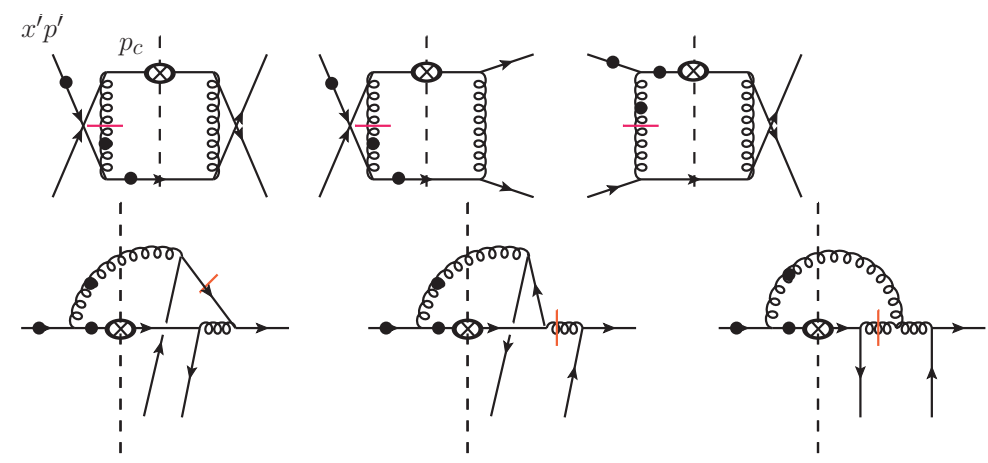

Figure 2: Lowest order diagrams for the hard part of the SFP contribution in the $q q^{\prime} \rightarrow q q^{\prime}$ and $q q \rightarrow q q$ channels. The twist-3 distribution contributes from the lower side of each diagram. For each diagram, three diagrams corresponding to a different attachment of the coherent gluon line to one of the dots need to be considered. The barred propagator gives rise to SFP. Mirror diagrams also contribute.
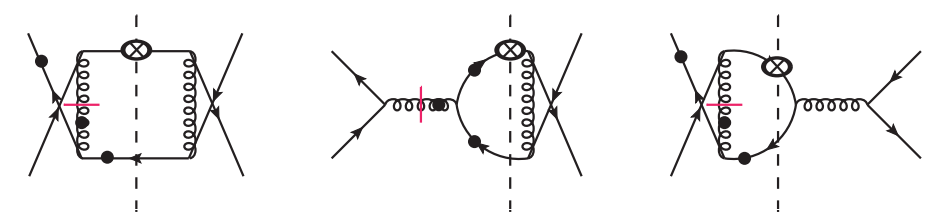

Figure 3: The same as Fig. 2, but for the $q \bar{q} \rightarrow q \bar{q}$ channel. Diagrams for the $\bar{q} q \rightarrow q \bar{q}$ channel are obtained by reversing the arrows of the quark lines and shifting the fragmentation insertion to the other quark line crossing the final-state cut.
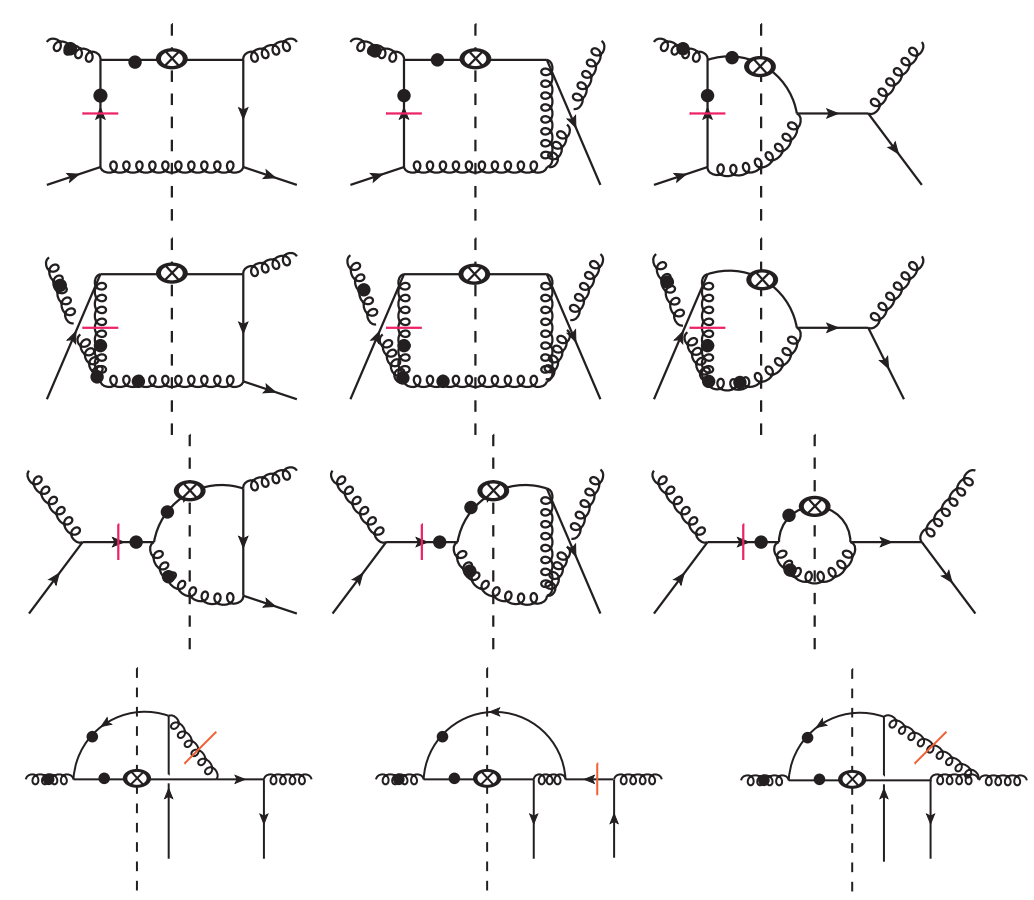

Figure 4: The same as Fig.2, but for the $q g \rightarrow q g$ channel. 


\section{Summary}

In this study, we calculated the contribution from the twist-3 quark-gluon correlation function in the unpolarized nucleon to $p p \rightarrow \Lambda^{\uparrow} X$. For the SGP contribution, we have developed the master formula and have confirmed the previous result which was obtained by direct calculation. The master formula is useful to understand why only the derivative term survive and also to include next-to-leading-order correction to the SGP cross section. We have also calculated the SFP contribution and have shown that it vanishes identically in all channels. Accordingly the derivative term of the SGP contribution is the only term for the twist-3 distribution contribution to $p p \rightarrow \Lambda^{\uparrow} X$. For the analysis of the hyperon polarization one needs to include the contribution from the twist-3 fragmentation function, which will be reported elsewhere.

\section{Acknowledgments}

This work has been supported by the Grant-in-Aid for Scientific Research from the Japanese Society of Promotion of Science under Contract No. 26287040 (Y. K. and S. Y.).

\section{References}

[1] G. Bunce et al., Phys. Rev. Lett. 36, 1113 (1976).

[2] J. w. Qiu and G. F. Sterman, Phys. Rev. Lett. 67, 2264 (1991).

[3] H. Eguchi, Y. Koike and K. Tanaka, Nucl. Phys. B 752, (2006) [arXiv:hep-ph/0604003].

[4] J. w. Qiu and G. F. Sterman, Phys. Rev. D 59, 014004 (1999) [hep-ph/9806356].

[5] Y. Kanazawa and Y. Koike, Phys. Lett. B 478, 121 (2000) [hep-ph/0001021].

[6] C. Kouvaris, J. W. Qiu, W. Vogelsang and F. Yuan, Phys. Rev. D 74, 114013 (2006) [hep-ph/0609238].

[7] Y. Koike and T. Tomita, Phys. Lett. B 675, 181 (2009) [arXiv:0903.1923 [hep-ph]].

[8] A. Metz and D. Pitonyak, Phys. Lett. B 723, 365 (2013) [arXiv:1212.5037 [hep-ph]].

[9] H. Beppu, K. Kanazawa, Y. Koike and S. Yoshida, Phys. Rev. D 89, no. 3, 034029 (2014) [arXiv:1312.6862 [hep-ph]].

[10] J. Adams et al. [STAR Collaboration], Phys. Rev. Lett. 92, 171801 (2004) [hep-ex/0310058]; B. I. Abelev et al. [STAR Collaboration], Phys. Rev. Lett. 101, 222001 (2008) [arXiv:0801.2990 [hep-ex]].

[11] K. Kanazawa, Y. Koike, A. Metz and D. Pitonyak, Phys. Rev. D 89, no. 11, 111501 (2014) [arXiv:1404.1033 [hep-ph]].

[12] Y. Kanazawa and Y. Koike, Phys. Rev. D 64, 034019 (2001) [hep-ph/0012225].

[13] J. Zhou, F. Yuan and Z.-T. Liang, Phys. Rev. D 78, 114008 (2008). [arXiv:0808.3629[hep-ph]]

[14] Y. Koike and K. Tanaka, Phys. Lett. B 646, 232 (2007) [Erratum-ibid. B 668, 458 (2008)] [hep-ph/0612117].

[15] Y. Koike and K. Tanaka, Phys. Rev. D 76, 011502 (2007) [hep-ph/0703169].

[16] Y. Koike, K. Yabe and S. Yoshida, Phys. Rev. D 92, no. 9, 094011 (2015) [arXiv:1509.06830 [hep-ph]]. 